Central European Journal of Energetic Materials, 2016, 13(1), 149-159

ISSN 1733-7178

e-ISSN 2353-1843

\title{
Thermal Decomposition Kinetics of Hexanitrohexaazaisowurtzitane/Ammonium Perchlorate
}

Yan-Li ZHU*, Kang-Kang WANG, Ming-Xin SHAN, Xiao-Di ZHENG, Qing-Jie JIAO, Jing-Si WANG

State Key Laboratory of Explosive Science and Technology, Beijing Institute of Technology,

South Street 5, Zhongguancun, Haidian District, Beijing, 100081, China

"E-mail: zhuyanli1999@bit.edu.cn

\begin{abstract}
The thermal decomposition kinetics of hexanitrohexaazaisowurtzitane/ ammonium perchlorate (HNIW/AP) have been investigated by thermogravimetrydifferential scanning calorimetry-mass spectrometry (TG-DSC-MS) simultaneous analysis. TG showed that there were three weight loss processes for the thermal decomposition of the HNIW/AP mixture. The first was ascribed mainly to the thermal decomposition of HNIW, while the second and third were assigned to that of AP. The presence of AP has little effect on the thermal decomposition process of the HNIW component. The apparent activation energy of the thermal decomposition of the HNIW component, calculated by the Kissinger method, was little changed compared to that of neat HNIW. The addition of HNIW to AP caused the onset and end temperatures of the thermal decomposition to be decreased and the decomposition process to be shortened. The high-temperature and lowtemperature decomposition processes of AP became blurred in the presence of HNIW, and this was supported by the MS results.
\end{abstract}

Keywords: hexanitrohexaazaisowurtzitane, ammonium perchlorate, thermal analysis, thermal decomposition kinetics

\section{Introduction}

2,4,6,8,10,12-Hexanitro-2,4,6,8,10,12-hexaazaisowurtzitane (HNIW, CL-20) is a newly developed caged nitramine, which has a greater density, good 
chemical stability and better performance than cyclotrimethylenetrinitramine (RDX) and cyclotetramethylenetetranitramine (HMX) [1]. Ammonium perchlorate (AP) is the most common oxidizer in various propellant, explosive and pyrotechnic compositions [2]. It is predicted that HNIW/AP could be a substitute for HMX/AP or RDX/AP, which have been extensively prepared as explosive or propellant compositions [3]. Thermal behaviour has a serious effect on material macroscopic properties. Therefore, the thermal decomposition characteristics of HNIW/AP have to be studied systematically before it can be used widely.

Many papers have been published concerning the thermal decomposition of neat HNIW [4-6] or AP [7-10]. However, few have addressed the thermal decomposition and reaction kinetics of the HNIW/AP mixture. Ding and her co-workers [5] studied the thermal decomposition characteristics of HNIW and AP in NEPE propellant by thermogravimetry (TG). The results showed that the decomposition temperature of AP was decreased because of the decomposition product of HNIW, while the presence of AP had little effect on the thermal decomposition process of HNIW. Bohn [6] investigated the thermal ageing of rocket propellant formulations containing HNIW and AP by heat generation rate and mass loss. However, no research has been related to the thermal decomposition kinetics of HNIW/AP mixtures. The performance of the explosives or propellants is highly relevant to the interaction between HNIW and AP. In this paper, the thermal decomposition characteristics of HNIW/AP were examined with the aid of a set of experimental devices, differential scanning calorimetry (DSC) - TG - mass spectrometry (MS).

\section{Materials and Methods}

\subsection{Materials and characterization}

Raw HNIW (Qingyang Chemical Industry Corporation, Liaoning Province, China) was recrystallized using ethyl acetate (analytical grade, Tianjin Chemical Work, Tianjin, China) as the solvent and petroleum ether (analytical grade, Tianjin Chemical Work, Tianjin, China) as the anti-solvent at ambient temperature. Details of the preparation procedures can be found elsewhere [13]. The average crystal size of the products was $80 \mu \mathrm{m}$ and the polymorph was confirmed to be $\varepsilon$-HNIW by the results of a field emission scanning electron microscope (FESEM, Hitachi S-4700, Japan) and X-ray diffraction (XRD, Bruker D4, Cu K $\alpha$ ). AP (Dalian Potassium Chlorate Works, Dalian, China, >99\%) was analytical grade with an average particle size of $30-40 \mu \mathrm{m}$. Mixtures of HNIW and AP 
powders were prepared by mixing the two components in a polished carnelian mortar for $1 \mathrm{~h}$. The mass ratio of HNIW to AP was 1:1 or 1:2.

\subsection{Thermal analysis}

A Netzsch STA449C (Selb, Germany) and Netzsch-QMS403C (Selb, Germany) were used for thermal analysis. The ionizing electron energy of the NetzschQMS403C was $70 \mathrm{eV}$. The inject ion pressure of the quartz capillary gas connector was 1 bar and the capillary temperature was $200^{\circ} \mathrm{C}$. The gas cell and gas tube between the TG-DSC and MS remained at $200{ }^{\circ} \mathrm{C}$.

A $~ 1 \mathrm{mg}$ sample was placed in an alumina crucible with a pin-hole in the lid and heated from 30 to $500{ }^{\circ} \mathrm{C} . \alpha-\mathrm{Al}_{2} \mathrm{O}_{3}$ was used as the reference sample. High-purity argon was used with a gas flow rate of $20 \mathrm{~mL} \cdot \mathrm{min}^{-1}$.

Non-isothermal thermal decomposition of HNIW/AP was performed at heating rates of $5,10,15$ and $20^{\circ} \mathrm{C} \cdot \mathrm{min}^{-1}$. The Kissinger and Flynn-Wall-Ozawa methods were used to obtain kinetic parameters based on the non-isothermal data [12]. The thermal decomposition process of the HNIW/AP mixture was monitored by MS on-line at a heating rate of $10^{\circ} \mathrm{C} \cdot \mathrm{min}^{-1}$.

\section{Results and Discussion}

\subsection{TG-DSC}

The TG-DSC curves of neat HNIW, HNIW/AP (1:1), HNIW/AP (1:2) and neat $\mathrm{AP}$ at a heating rate of $10{ }^{\circ} \mathrm{C} \cdot \mathrm{min}^{-1}$ are shown in Figures 1 and 2. Figure 1 shows that there is a single mass loss process for neat HNIW, two for AP and three for the HNIW/AP mixtures. The first mass loss step for the HNIW/AP mixtures was ascribed to the thermal decomposition of HNIW since the onset and end temperatures of the first mass loss of the HNIW/AP mixtures were at about 229 and $255^{\circ} \mathrm{C}$, respectively, which are almost the same as those of neat HNIW [14]. The second and third mass loss steps were caused by the low-temperature decomposition (LTD) and high-temperature decomposition (HTD) of AP, respectively [15]. The onset and end temperatures of these two steps had decreased compared to those of neat AP, suggesting that the thermal decomposition process of AP is catalyzed by the presence of HNIW. The related temperatures of the thermal decomposition of the various samples are summarized in Table 1.

Figure 2 shows that the first exothermic decomposition peak of the HNIW component was at about $249^{\circ} \mathrm{C}$, and had changed little compared to neat HNIW. The addition of AP to HNIW caused a relatively strong decrease in the exothermic 
peak area, resulting from the decrease in the mass ratio of HNIW in the mixture.

The DSC curve of neat AP showed that there was an endothermic peak temperature of about $244{ }^{\circ} \mathrm{C}$, which was ascribed to the transition from the orthorhombic to the cubic phase since there was no change in the mass of AP $[16,17]$. The addition of HNIW to AP caused the endothermic peak to become weaker. The position of the endothermic peak of HNIW/AP (1:2) had shifted to $242{ }^{\circ} \mathrm{C}$, and was hard to distinguish in the case of HNIW/AP (1:1) since the endothermic peak of AP was covered by the exothermic peak of HNIW. Two exotherms with maximum peak temperatures of $309^{\circ} \mathrm{C}$ and $350^{\circ} \mathrm{C}$ respectively are assignable to the LTD and HTD of AP, corresponding to the two mass loss steps in the TG curve. The onset temperature of the LTD process of neat AP was $285^{\circ} \mathrm{C}$, and shifted to $282{ }^{\circ} \mathrm{C}$ and $277^{\circ} \mathrm{C}$ for HNIW/AP $(1: 2)$ and HNIW/AP (1:1), respectively, as shown in Figure 2. The second exothermic peak of AP became weaker for the HNIW/AP mixture and was covered by the first peak compared to neat AP. The LTD and HTD steps of AP became blurred in the presence of HNIW, and the end temperature of the thermal decomposition of AP decreased in the DSC curves, as tabulated in Table 1, suggesting a catalytic effect of HNIW on AP.

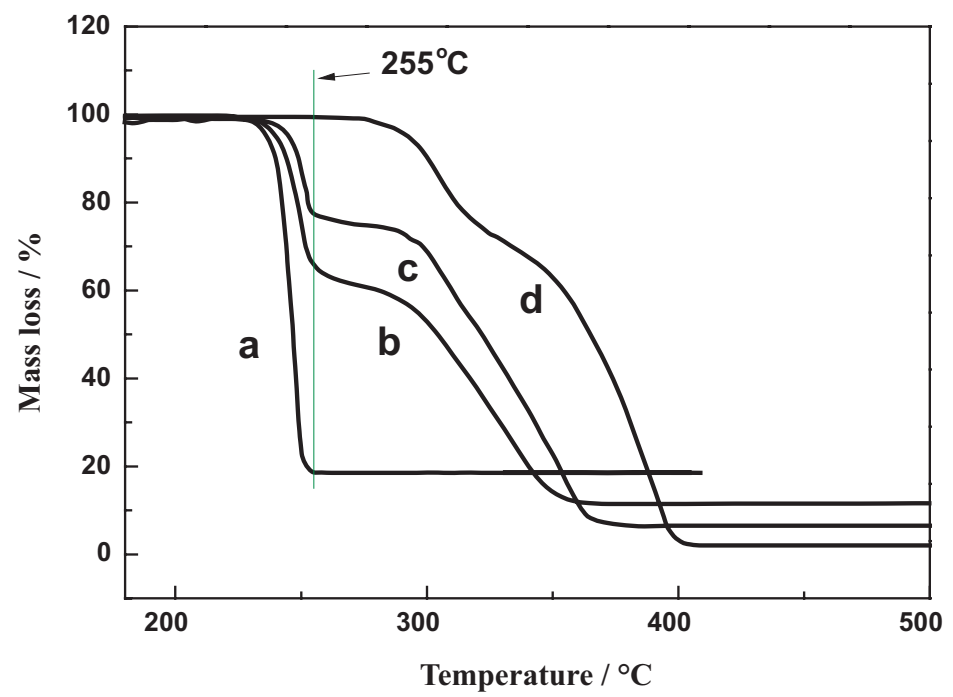

Figure 1. TG curves of HNIW (a), HNIW/AP (1:1) (b), HNIW/AP (1:2) (c) and $\mathrm{AP}(\mathrm{d})$ at heating rate of $10^{\circ} \mathrm{C} \cdot \mathrm{min}^{-1}$. 


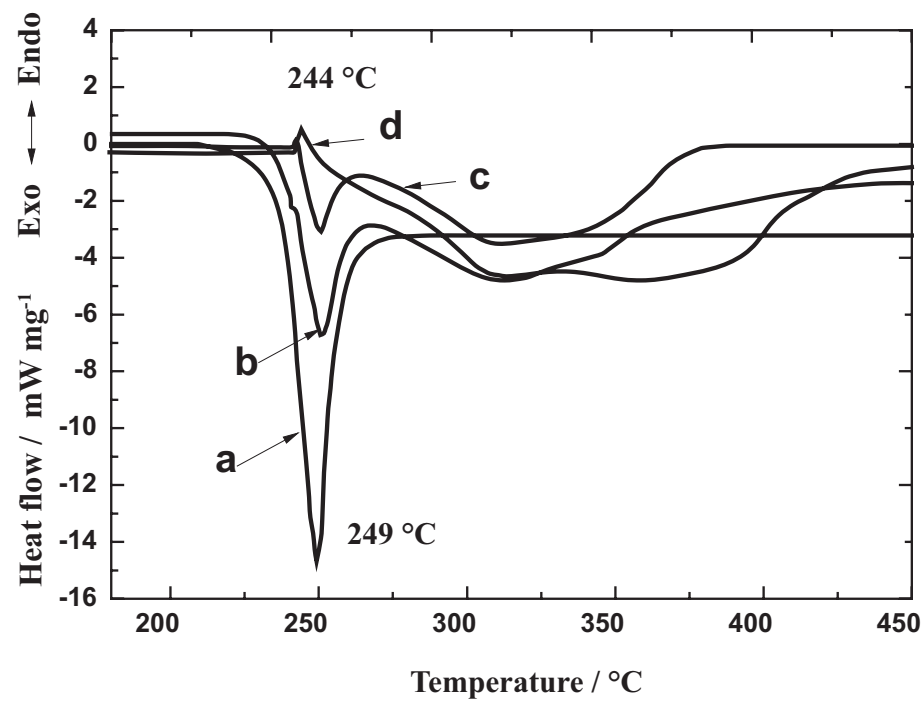

Figure 2. DSC curves of HNIW (a), HNIW/AP (1:1) (b), HNIW/AP (1:2) (c) and $\mathrm{AP}(\mathrm{d})$ at heating rate of $10^{\circ} \mathrm{C} \cdot \mathrm{min}^{-1}$.

Table 1. Temperature and kinetic parameters of the thermal decomposition of HNIW and AP at $10^{\circ} \mathrm{C} \cdot \mathrm{min}^{-1}$

\begin{tabular}{|c|c|c|c|c|c|c|c|c|}
\hline \multicolumn{3}{|c|}{ Composition } & $\begin{array}{c}T_{o} \\
{\left[{ }^{\circ} \mathrm{C}\right]}\end{array}$ & $\begin{array}{c}T_{p} \\
{\left[{ }^{\circ} \mathrm{C}\right]}\end{array}$ & $\begin{array}{c}T_{e} \\
{\left[{ }^{\circ} \mathrm{C}\right]}\end{array}$ & $\begin{array}{c}E_{K} \\
{\left[\mathrm{~kJ} \cdot \mathrm{mol}^{-1}\right]}\end{array}$ & $\lg \left(A_{K} / s^{-1}\right)$ & $\gamma_{K}$ \\
\hline \multicolumn{3}{|c|}{ HNIW } & 227 & 249 & 255 & 174.6 & 17.19 & 0.997 \\
\hline \multirow{3}{*}{$\begin{array}{l}\text { HNIW/AP } \\
(1: 1)\end{array}$} & \multicolumn{2}{|c|}{ HNIW } & 229 & 250 & 255 & 171.1 & 16.97 & 0.998 \\
\hline & \multirow{2}{*}{ AP } & LTD & 277 & 311 & - & 143.5 & 14.22 & 0.999 \\
\hline & & HTD & - & 346 & 353 & 121.6 & 10.49 & 0.996 \\
\hline \multirow{3}{*}{$\begin{array}{l}\text { HNIW/AP } \\
(1: 2)\end{array}$} & \multicolumn{2}{|c|}{ HNIW } & 231 & 250 & 255 & 158.9 & 15.70 & 0.981 \\
\hline & \multirow{2}{*}{ AP } & LTD & 282 & 310 & - & 135.7 & 12.09 & 0.991 \\
\hline & & HTD & - & 346 & 366 & 123.4 & 10.89 & 0.999 \\
\hline \multirow{2}{*}{ AP } & \multicolumn{2}{|c|}{ LTD } & 285 & 309 & 316 & 123.7 & 10.63 & 0.998 \\
\hline & \multicolumn{2}{|c|}{ HTD } & 344 & 350 & 399 & 132.4 & 11.58 & 0.997 \\
\hline
\end{tabular}

$T_{o}$ - onset temperature of thermal decomposition, $T_{e}$ - end temperature of thermal decomposition, $T_{p}$ - peak temperature of thermal decomposition, $E_{K}-$ apparent activation energy, $A_{k}-$ pre-exponential factor, $\gamma_{K}$ - linear correlation coefficient.

\subsection{Non-isothermal analysis}

Figures 3 and 4 show the TG-DSC curves of the HNIW/AP (1:1) mixture at heating rates of $5,10,15$ and $20^{\circ} \mathrm{C} \cdot \mathrm{min}^{-1}$. The onset, peak and end temperatures of the thermal decomposition processes of the mixture increased with increasing 
heating rate. Similar trends (not shown here) were also obtained for neat HNIW and AP.

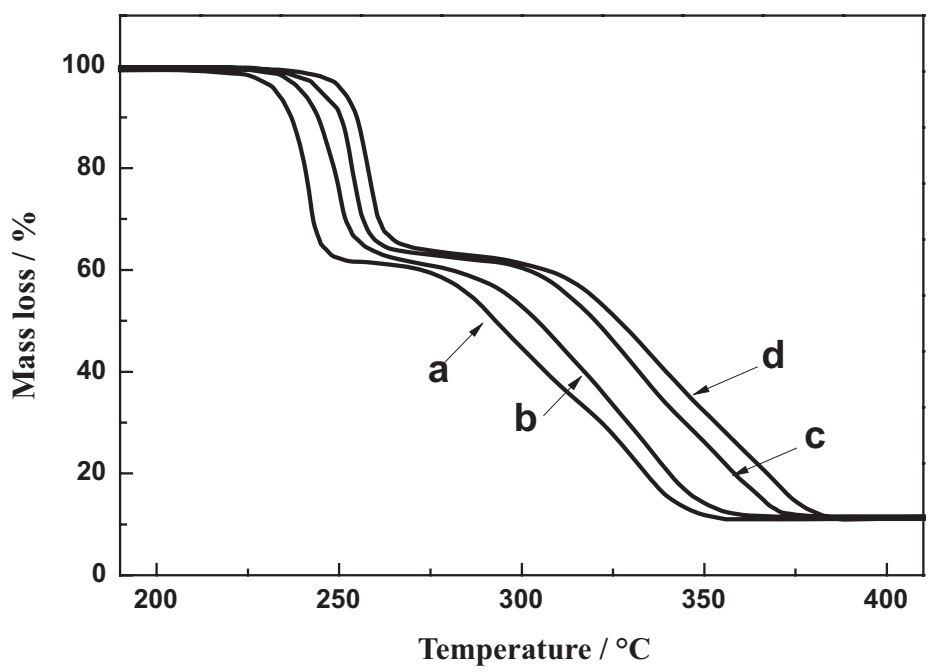

Figure 3. TG curves of HNIW/AP $(1: 1)$ at heating rates of $5{ }^{\circ} \mathrm{C} \cdot \mathrm{min}^{-1}$ (a), $10{ }^{\circ} \mathrm{C} \cdot \mathrm{min}^{-1}(\mathrm{~b}), 15^{\circ} \mathrm{C} \cdot \mathrm{min}^{-1}(\mathrm{c})$ and $20^{\circ} \mathrm{C} \cdot \mathrm{min}^{-1}(\mathrm{~d})$.

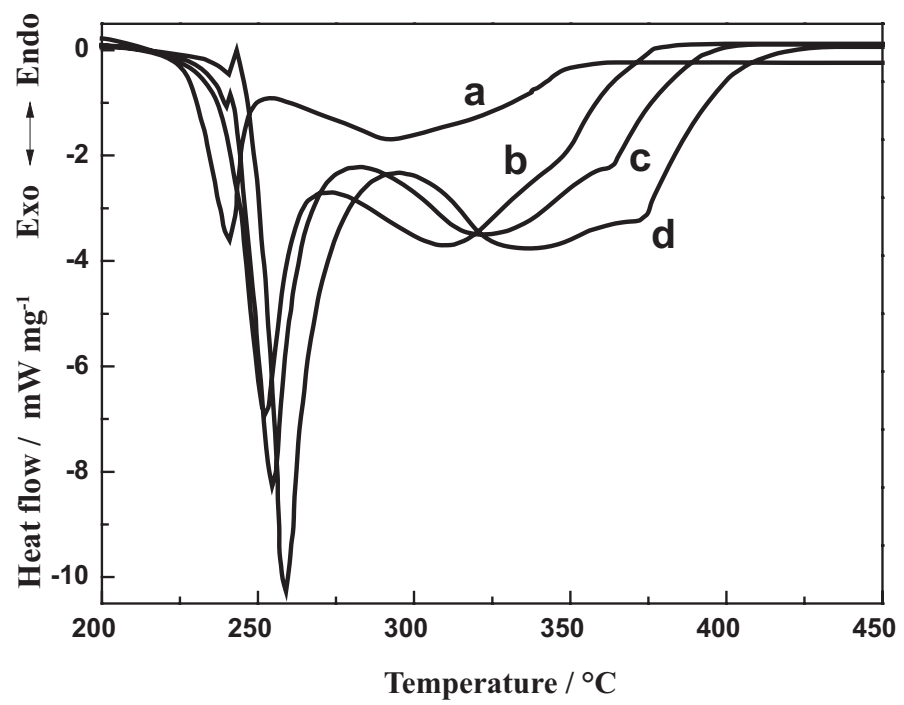

Figure 4. DSC curves of $\operatorname{HNIW/AP}(1: 1)$ at heating rates of $5{ }^{\circ} \mathrm{C} \cdot \mathrm{min}^{-1}$ (a), $10{ }^{\circ} \mathrm{C} \cdot \mathrm{min}^{-1}(\mathrm{~b}), 15^{\circ} \mathrm{C} \cdot \mathrm{min}^{-1}(\mathrm{c})$ and $20^{\circ} \mathrm{C} \cdot \mathrm{min}^{-1}(\mathrm{~d})$. 
The Kissinger method [18] was used to calculate the apparent activation energy $E_{K}$ and pre-exponential factor $A$ based on the data in Figure 3. The activation energies of other samples were also calculated by the same method, as shown in Table 1, and are close to the activation energy $E_{o}$ obtained by the Flynn-Wall-Ozawa method [19].

The values of $E_{\alpha, i}$ at various conversion degrees $\alpha_{i}$ (conversion degree is the mass ratio of the reacted substance to the starting material) calculated by the Ozawa method show that the value of $E$, which is about $160 \mathrm{~kJ} \cdot \mathrm{mol}^{-1}$, has changed little in the range $0.1<\alpha<0.4$. Therefore, it was reasonable to investigate the decomposition mechanism in this range or the thermal decomposition of the HNIW component.

Five types of methods (Ordinary integral [20], MacCallum-Tanner [21], Coats-Redfern [22] and Šatava-Sesták [23] and Agrawal [24]) were introduced to determine the most probable kinetic model function. Forty one types of kinetic model functions [20] and the data $\alpha_{i}$ and $T_{i}$ from the TG-DTG curves at different heating rates from Figure 3 were substituted into the above five equations to calculate the values of $E, A, \gamma$ and $Q$ (standard mean square deviation). Some statistical criteria, such as $\gamma$ and $Q$, were applied to decide the most probable mechanism function, $G(\alpha)$. The values of $A$ and $E$ obtained by the Kissinger method were also applied to evaluate the validity of $G(\alpha)$.

The results indicated that the first mass loss step, or the thermal decomposition process of the HNIW component in the HNIW/AP (1:1) mixture, can be described by an Avrami-Erofeev equation with $G(\alpha)=[-\ln (1-\alpha)]^{1 / 3}$ or $f(\alpha)=3(1-\alpha)[-$ $\ln (1-\alpha)]^{2 / 3}$, which is the same as that of neat HNIW, suggesting that this step is controlled by nucleation and subsequent growth. It may be inferred that AP has little effect on the thermal decomposition of HNIW.

\subsection{MS analysis}

The non-isothermal, thermal decomposition processes of HNIW, HNIW/AP (1:1), HNIW/AP (1:2) and neat AP were monitored by MS on-line at a heating rate of $10^{\circ} \mathrm{C} \cdot \mathrm{min}^{-1}$. The results showed that although the ion current intensities of each gas product were different, all of the mixtures had the same primary ion current numbers $(\mathrm{m} / \mathrm{z})$ of $16,17,18,28,30,32$ and 44 . The possible assignments of these are $\mathrm{CH}_{4}{ }^{+} / \mathrm{NH}_{2}{ }^{+}, \mathrm{NH}_{3}{ }^{+} / \mathrm{OH}^{+}, \mathrm{H}_{2} \mathrm{O} / \mathrm{NH}_{4}{ }^{+}, \mathrm{CO}^{+} / \mathrm{N}_{2}{ }^{+} / \mathrm{H}_{2} \mathrm{CN}^{+}, \mathrm{NO}^{+} / \mathrm{CH}_{2} \mathrm{O}^{+}$, $\mathrm{C}_{2} \mathrm{H}_{6} / \mathrm{N}_{2} \mathrm{H}_{2}$ and $\mathrm{CO}_{2}{ }^{+} / \mathrm{N}_{2} \mathrm{O}^{+} / \mathrm{C}_{2} \mathrm{H}_{4} \mathrm{O}$. Figures 5 and 6 showed the mass spectra of the gaseous products (with $\mathrm{m} / \mathrm{z}$ values of 18 and 44 ) from the decomposition of HNIW, HNIW/AP (1:1), HNIW/AP (1:2) and neat AP. The onset, peak and end temperatures for $\mathrm{m} / \mathrm{z} 18$ and 44 of the various samples are summarized in Table 2 . 


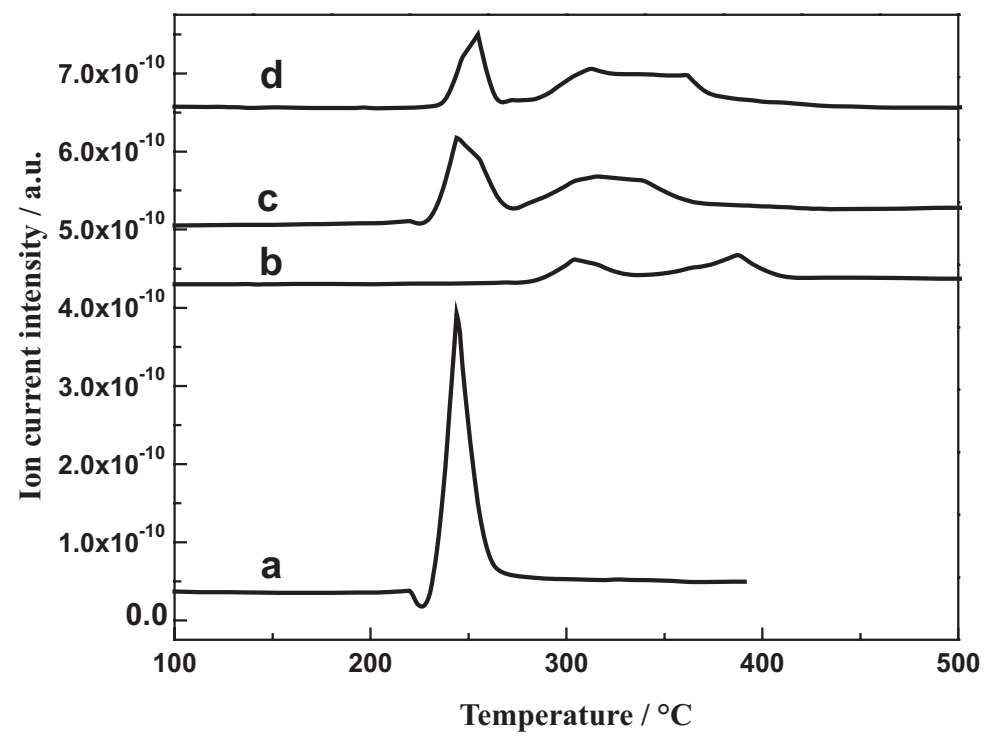

Figure 5. MS of m/z 18 of HNIW (a), AP (b), HNIW/AP (1:1) (c) and HNIW/AP $(1: 2)(d)$ at a heating rate of $10{ }^{\circ} \mathrm{C} \cdot \mathrm{min}^{-1}$.

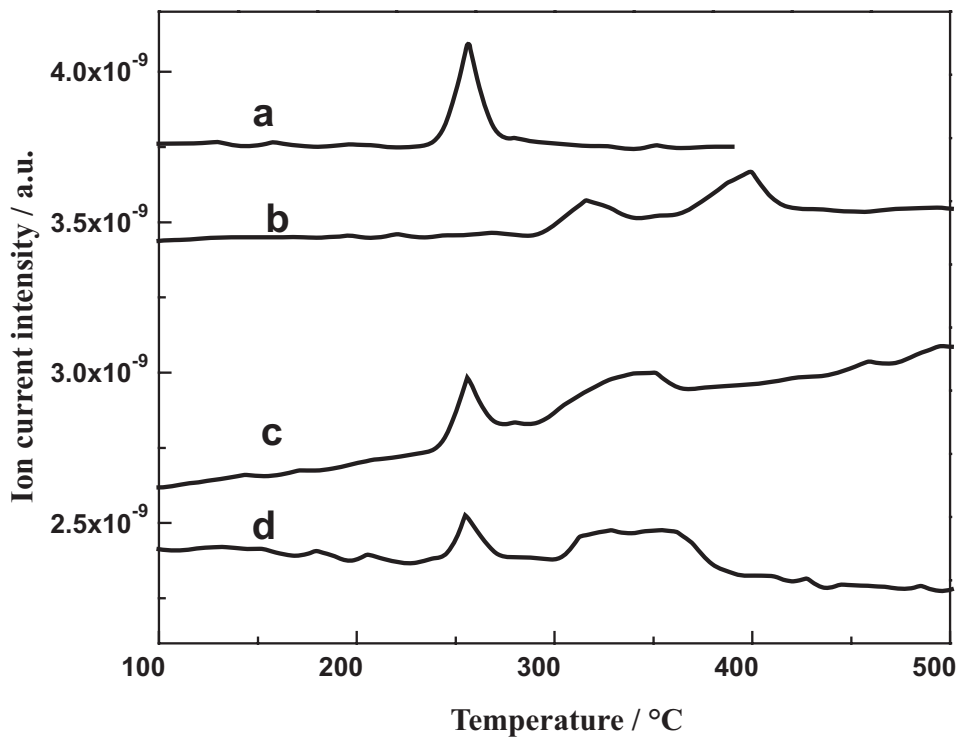

Figure 6. MS of m/z 44 of HNIW (a), AP (b), HNIW/AP (1:1) (c) and HNIW/AP (1:2) (d) at a heating rate of $10^{\circ} \mathrm{C} \cdot \mathrm{min}^{-1}$. 
Table 2. The corresponding temperatures $\left(T_{o}-T_{p}-T_{e}\right)$ of $\mathrm{m} / \mathrm{z} 18$ and 44

\begin{tabular}{|c|c|c|c|c|c|c|c|}
\hline \multirow[b]{2}{*}{$\mathrm{m} / \mathrm{z}$} & \multirow[b]{2}{*}{$\begin{array}{l}\text { HNIW } \\
T_{o}-T_{p}-T_{e}\end{array}$} & \multicolumn{2}{|c|}{ HNIW/AP (1:1) } & \multicolumn{2}{|c|}{ HNIW/AP (1:2) } & \multicolumn{2}{|c|}{$\mathrm{D}$} \\
\hline & & $\begin{array}{c}\text { HNIW } \\
T_{o}-T_{p}-T_{e}\end{array}$ & $\begin{array}{c}\mathrm{AP} \\
T_{0}-T_{e}\end{array}$ & $\begin{array}{c}\text { HNIW } \\
T_{o}-T_{p}-T_{e}\end{array}$ & $\begin{array}{c}\mathrm{AP} \\
T_{o}-T_{e}\end{array}$ & $\begin{array}{c}\text { LTD } \\
T_{o}-T_{p}-T_{e}\end{array}$ & $\begin{array}{c}\text { HTD } \\
T_{o}-T_{p}-T_{e}\end{array}$ \\
\hline 18 & & $242-256-275$ & $290-366$ & $242-256-276$ & $301-395$ & $292-316-352$ & $362-399-427$ \\
\hline 44 & 27-244-267 & $229-244-274$ & $272-370$ & $233-244-267$ & $281-398$ & $282-304-338$ & $338-387-415$ \\
\hline
\end{tabular}

The onset temperatures for the various samples detected by MS were higher than those obtained from the TG-DSC results by about $10^{\circ} \mathrm{C}$, as shown in Tables 1 and 2, probably due to the gas tube between the TG-DSC and the MS spectrometer. After the addition AP to HNIW, the ion current numbers had changed little, while the intensity of the gaseous products produced from the HNIW thermal decomposition had decreased, which is similar to the results obtained from the DSC curves in Figure 2. A decrease in the exothermic peak area caused by the HNIW thermal decomposition was observed after the addition AP to HNIW. The ion current intensity of each gas product produced two peaks for neat AP, corresponding to the LTD and HTD processes. These two processes gradually merged into one on increasing the amount of HNIW, as shown in Figures 5 and 6 . The onset temperatures for almost all of the ions decreased, and the AP decomposition process became shortened in the presence of HNIW, suggesting a catalytic effect of HNIW on AP decomposition.

\section{Conclusions}

The thermal decomposition of HNIW/AP mixtures was studied by TG-DSC-MS. The TG-DSC study showed that the thermal decomposition of HNIW was hardly affected by the presence of AP. The non-isothermal kinetics results showed that the thermal decomposition reaction mechanism function for the HNIW component of the HNIW/AP (1:1) mixture conformed to the Avrami-Erofeev equation, with the factor of nucleus growth $n=1 / 3$, which is the same as that of neat HNIW. The LTD and HTD processes of neat AP gradually merged into one with increasing amounts of HNIW. The onset and end temperatures of AP decreased in the presence of HNIW, suggesting a catalytic effect of HNIW on AP decomposition. These results were supported by the MS results.

\section{Acknowledgments}

The authors appreciate the financial support from the National Natural Science Foundation of China (Grant Nos. 51304024 and 11172042) and the Independent 
Research Subject of State Key Laboratory of Explosion Science and Technology (Grant No. YBKT15-04).

\section{References}

[1] Yang R.J., An H.M., Tan H.M., Combustion and Thermal Decomposition of HNIW and HTPB/HNIW Propellants with Additives, Combust. Flame, 2003, 135, 463-473.

[2] Rajic M., Suceska M., Study of Thermal Decomposition Kinetics of Lowtemperature Reaction of Ammonium Perchlorate by Isothermal TG, J. Therm. Anal. Calorim., 2001, 63, 375-386.

[3] Gogulya M.F., Makhov M.N., Dolgoborodov A.Y., Mechanical Sensitivity and Detonation Parameters of Aluminized Explosives, Combust Explosion Shock Waves (Engl. Transl.), 2004, 40(4), 445-457.

[4] Naika N.H., Gorea G.M., Gandheb B.R., Sikder A.K., Studies on Thermal Decomposition Mechanism of CL-20 by Pyrolysis Gas Chromatography-Mass Spectrometry (Py-GC/MS), J. Hazard. Mater, 2008, 159, 630-635.

[5] Ding L., Zhao F.Q., Liu Z.R., Zhang L.Y., Heng S.Y., The Interaction Between the Components in NEPE Propellant Containing CL-20 (in Chinese), Chinese Journal of Explosives \& Propellants, 2008, 31(2), 38-42.

[6] Bohn M.A., Thermal Ageing of Rocket Propellant Formulations Containing $\varepsilon$-HNIW ( $\varepsilon$-CL20) Investigated by Heat Generation Rate and Mass Loss, Thermochim. Acta, 2003, 401, 27-41.

[7] Liu R., Zhou Z., Yin Y., Yang L., Zhang T., Dynamic Vacuum Stability Test Method and Investigation on Vacuum Thermal Decomposition of HMX and CL20, Thermochim. Acta, 2012, 537, 13-19.

[8] Patil D.G., Brill T.B., Thermal Decomposition of Energetic Materials 53. Kinetics and Mechanism of Thermolysis of Hexanitrohexazaisowurtzitane, Combust. Flame, 1991, 87, 145-151.

[9] Liu L., Li F., Tan L., Ming L., Yi Y., Effects of Nanometer Ni, Cu, Al and NiCu Powders on the Thermal Decomposition of Ammonium Perchlorate, Propellants Explos. Pyrotech., 2004, 29(1), 34-38.

[10] Zhi J., Wang T., Li S., Zhao F., Liu Z., Yang C., Yang L., Liu S., Zhang G., Thermal Behavior of Ammonium Perchlorate and Metal Powders of Different Grades, J. Therm. Anal. Calorim., 2006, 85, 315-320.

[11] Liu Z.R., Thermal Analysis of Energetic Materials (in Chinese), National Defence Industry Press, Beijing, 2008, ISBN: 9787118059724.

[12] Ren Y.L., Cheng B.W., Zhang J.S., Jiang A.B., Fu W.L., Thermal Degradation Kinetics of N,N'-Di(diethoxythiophosphoryl)-1,4-phenylenediamine, Chem. Res. Chinese Universities, 2008, 24(5), 628-631.

[13] Jiang X.B., Guo X.Y., Ren H., Zhu Y.L., Jiao Q.J., Control of Particle Size and Shape of $\varepsilon$-HNIW in Drowning-out Crystallization, J. Chem. Eng. Jpn., 2012, 
45(6), 380-386.

[14] Löbbecke S., Bohn M.A., Pfeil A., Krause H., Thermal Behavior and Stability of HNIW (CL-20), $29^{\text {th }}$ Int. Annu. Conf. ICT, Karlsruhe, Germany, 1998, 145.

[15] Singh G., Felix S.P., Soni P., Studies on Energetic Compounds: Part 31. Thermolysis and Kinetics of RDX and Some of its Plastic Bonded Explosives, Thermochim. Acta, 2005, 426, 131-139.

[16] Shen S., Wu B., The Thermal Decomposition of Ammonium Perchlorate (AP) Containing a Burning-rate Modifier, Thermochim. Acta, 1993, 223, 135-143.

[17] Jiao Q.J., Zhu Y.L., Huang H., Ren H, Thermal Decomposition of RDX/AP by TG-DSC-MS-FTIR, J. Therm. Anal. Calorim., 2014, 116, 1125-1131.

[18] Kissinger H.E., Reaction Kinetics in Differential Thermal Analysis, Anal. Chem., 1957, 29, 1702-1706.

[19] Ozawa T., Kinetic Analysis of Derivative Curves in Thermal Analysis, J. Therm. Anal. Calorim., 1970, 2, 301-324.

[20] Hu R.Z., Gao S.L., Zhao F.Q., Shi Q.Z., Zhang T.L., Zhang J.J., Thermal Analysis Kinetics (in Chinese), $2^{\text {nd }}$ ed., Science Press, Beijing, 2008, ISBN: 7-03-009463/O.1511.

[21] MacCallum J.R., Tanner J., A Comparative Study of Some Methods of Assessing Kinetic Parameters from Thermogravimetric Analysis, Eur. Polym. J., 1970, 6, 907-917.

[22] Coats A.W., Redfern J.P., Kinetics Parameters from Thermaogravimetric Data, Nature, 1964, 201, 68-69.

[23] Šatava F., Šesták J., Computer Calculation of the Mechanism and Associated Kinetic Data Using a Non-isothermal Integral Method, J. Therm. Anal. Calorim., 1975, 8, 477-489.

[24] Agrawal R.K., A New Equation for Modeling Nonisothermal Reactions, J. Therm. Anal. Calorim., 1987, 32, 149-156. 
